\title{
Variabilité saisonnière et disponibilité des ressources alimentaires végétales consommées par les Mones de Lowe Cercopithecus lowei Thomas, 1923 dans la forêt de l'Université Nangui Abrogoua, Abidjan- Côte d'Ivoire
}

\author{
Sami Blaise $\operatorname{KAMBIRE}^{1,2 *}$, Karim OUATTARA ${ }^{1,2}$, \\ Jean Louis KOUAKOU ${ }^{1,3}$ et Inza KONE KO,2 $^{1,2}$ \\ ${ }^{1}$ Laboratoire des Milieux naturels et Conservation de la Biodiversité, UFR Biosciences, Université Felix \\ Houphouët Boigny-Abidjan, 22 BP 582 Abidjan 22, Côte d'Ivoire. \\ ${ }^{2}$ Centre Suisse de Recherches Scientifiques en Côte d'Ivoire (CSRS), 01 BP 1303 Abidjan 01, Côte d'Ivoire. \\ ${ }^{3}$ Laboratoire de biotechnologie, Agriculture et Valorisation des Ressources Biologiques, UFR Biosciences, \\ Université Felix Houphouët Boigny-Abidjan, 22 BP 582 Abidjan 22, Côte d'Ivoire. \\ *Auteur correspondant, E-mail : sami.kambire@csrs.ci ; Tel : +2250709777793.
}

Received: 20-05-2021

Accepted: 20-10-2021

Published: $30-10-2021$

\section{RESUME}

L'Université Nangui Abrogoua située à Abidjan -Côte d'Ivoire abrite une relique forestière, dans laquelle vivent des Mones de Lowe Cercopithecus lowei Thomas, 1923. Cette espèce est décrite comme Vulnérable selon l'Union Internationale de la Conservation de la Nature. La survie de ces singes dépend de la disponibilité des ressources alimentaires. Dans un contexte de pressions de plus en plus grandissantes à cause de l'urbanisation, une étude a été entreprise pour évaluer la variabilité de la diversité et de la disponibilité des ressources alimentaires de ces singes. Des suivis continus journaliers des singes et phénologiques des arbres fruitiers menés dans les différents habitats de recherche de nourriture pendant 96 semaines ont permis de déterminer leur choix alimentaire. Il ressort que, sur les 426 espèces végétales identifiées sur le site de l'étude, 115 espèces appartenant à 46 familles et 79 genres sont consommées par cette population de Mone de lowe, avec une trentaine d'espèces majoritairement utilisées dans leur alimentation. Les fruits occupent 79,6\% sur les sept différents organes de plantes qui sont apparus comme les items les plus consommés. Ces items consommés sont plus inféodés à la strate végétale moyenne et localisés principalement dans la jachère que dans la forêt secondaire et les espaces cultivés. La disponibilité alimentaire des items consommés montre que les fruits sont plus abondants pendant les saisons pluvieuses. De plus, durant le cycle saisonnier, ces groupes de Mones de Lowe ont consommé préférentiellement 30 espèces végétales.

(C) 2021 International Formulae Group. All rights reserved.

Mots clés : Mone de Lowe, diversité végétale, préférence alimentaire, milieu urbanisé.

\section{Seasonal variability and availability of plant food resources consumed by the Mones of Lowe Cercopithecus lowei Thomas, 1923 in the forest of the University Nangui Abrogoua, Abidjan-Côte d'Ivoire}

\begin{abstract}
The University Nangui Abrogoua in Abidjan-Côte d'Ivoire homes a forest, in which live tree-tailed monkeys, Lowe's Monekey Cercopithecus lowei Thomas, 1923. This species is described as vulnerable by the International Union for Conservation of Nature. The survival of these monkeys is linked on the diversity of local
\end{abstract}


biological resources. In a context of increasing pressure from urbanization, this study was undertaken to assess the variability of diversity, availability, and palatability of food resources of these resilient monkeys. Continuous daily monitoring of monkeys and phenological monitoring of fruit trees in different foraging habitats over a 96weeks period determined their food choice. Of the 426 plant species identified at the study site, 115 species belonging to 46 families and 79 genera were consumed by this population of Lowe's monkeys, with about 30 species being the most used in their diet. Fruits occupied $79,6 \%$ of the seven different plant organs that emerged as the most consumed items. These consumed items are more related to the middle plant stratum and located mainly in the fallow than in the secondary forest and cultivated areas. Food availability of consumed items shows that fruits are more abundant during rainy seasons. In addition, during the seasonal cycle, these groups of Lowe's Mones preferentially consumed 30 plant species.

(C) 2021 International Formulae Group. All rights reserved.

Keywords: Lowe's monkey, plant diversity, food preference, urbanized environment.

\section{INTRODUCTION}

L'inquiétude concernant la survie des Primates non- humains $(\mathrm{PNH})$ forestiers est grandissante dans le monde (Estrada et al., 2017). En Afrique de l'Ouest, l'aire de répartition de la Mone de Lowe Cercopithecus lowei Thomas, 1923 se limite à la Côte d'Ivoire et le Ghana. Malheureusement, la menace d'extinction de cette espèce à l'instar des Primates est réelle car ses deux pays sont actuellement les premiers producteurs mondiaux de cacao (Bitty et al., 2015). Dans ces pays, la cacao culture itinérante se fait au détriment de la destruction de la forêt incluant la biodiversité qu'elle abrite (Asare et al., 2014). En effet, ces deux pays ont connu, depuis les années d'indépendance à nos jours, de fortes destructions de leurs couverts forestiers, qui abritaient, il $\mathrm{y}$ a quelques décennies une diversité spécifique de Primates (Bamba et al., 2017). A cela s'ajoute un taux d'urbanisation accéléré qui affecte la viabilité des espèces animales en général et des primates forestiers en particulier (Koné, 2015). Ainsi, l'urbanisation a restreint le domaine vital de ces espèces de Primates les contraignant à des adaptations alimentaires et sociales afin de garantir leur survie (Kone et al., 2011).

En Côte d'Ivoire, les populations sauvages et viables des Mones de Lowe se répartissent dans les aires protégées, à l'Est du fleuve Sassandra (Koné et al., 2011) mais aussi dans certaines forêts classées. Dans les domaines villageois comme les forêts sacrées, certains tabous locaux permettent la protection de cette espèce de singe (Bamba et al., 2017).
$\mathrm{Au}$ sud du pays, dans la capitale économique, l'exemple le plus emblématique est celui de la relique forestière de l'Université Nangui Abrogoua (UNA), séparée du Parc National du Banco, isolée au sein de ladite université, en plein cœur d'Abidjan qui abrite deux groupes de Mone de Lowe. Pour cette population résiduelle de la relique forestière de l'UNA, l'habitat s'est vu réduit avec les travaux d'extension de l'UNA en 2011-2012. Cette population isolée de l'UNA des autres Mones de Lowe de la forêt originelle du Parc National $\mathrm{du}$ Banco, a subi probablement subit des modifications comportementales du fait d'abord de cet isolement prolongé mais aussi de la proximité des hommes et des activités anthropiques. Ainsi, la survie de la population de Mones de Lowe dans cette relique forestière dépendrait de la diversité des ressources alimentaires notamment végétales disponibles. En effet, des études conduites sur les Mones de Campbell, espèce la plus proche de la mone de Lowe, montrent que cette espèce de singe présente un répertoire alimentaire composé de fruits, de feuilles, de racines, de fleurs, d'invertébrés, etc. (Buzzard, 2010). Ce régime alimentaire peut varier considérablement selon les saisons (Zausa et al., 2018). Face à la pression urbaine à laquelle fait face la Mone de Lowe de l'UNA, l'on s'interroge sur la qualité et la disponibilité végétale susceptibles de constituer les ressources alimentaires pour ces groupes en milieu urbain. Hormis l'étude relative à la stratégie de fourragement optimale testant la théorie de Charnov (Koné et al., 2011), aucune étude n'a été conduite sur ce 
groupe de Primates non humain. Ainsi, la présente étude qui a pour objectif général d'évaluer le répertoire alimentaire de ce groupe a été entreprise. De manière spécifique, il s'agit (i) d'identifier la diversité des espèces végétales consommées par ces individus de Mones et (ii) d'analyser la disponibilité saisonnière des items végétaux consommés et (iii) de déterminer la préférence alimentaire des végétaux consommés.

\section{MATERIEL ET METHODES Caractéristiques du milieu d'étude}

Le site d'étude est une forêt résiduelle, annexée à l'UNA à Abidjan-Côte d'Ivoire. Fragment du Parc National du Banco, elle est située entre $03^{\circ} 87^{\prime} 585 \mathrm{~N}$ et $05^{\circ} 95^{\prime} 866 \mathrm{~W}$ (Figure 1).

La superficie de cette relique forestière est estimée à 10 ha. Les différents habitats rencontrés sont principalement la jachère, une petite partie de forêt secondaire et des espaces cultivés dans lesquels fourragent les singes. Elle héberge une richesse floristique relativement importante. On y dénombre plus de 426 espèces réparties entre 259 genres et 60 familles (Missa et al., 2019). Comme la forêt $\mathrm{du}$ PNB, la relique forestière de l'UNA fait partie du secteur ombrophile ivoirien qui est placé sous un climat de type subéquatorial (Avenard et al.,1971 ; Lauginie, 2007). Le climat est caractérisé en général par deux facteurs qui sont la pluviométrie et la température. La moyenne mensuelle de température varie de $24,4{ }^{\circ} \mathrm{C}$ (août) à $27,88^{\circ} \mathrm{C}$ (mars) tandis que la précipitation moyenne maximale s'observe au mois de juin $(406,08$ $\mathrm{mm})$ et la valeur minimale au mois de janvier $(28,26 \mathrm{~mm})$ (Avenard et al.,1971 ; Lauginie, 2007). Les variations moyennes des précipitations de la ville d'Abidjan sont caractérisées par quatre saisons. Deux saisons pluvieuses et deux saisons sèches. Ainsi, la plus longue saison sèche s'étend de début janvier à fin février suivie par une plus longue saison des pluies, qui part du mois de mars jusqu'au mois de juillet. La saison courte sèche qui se situe au niveau du mois d'août, intervient après la grande saison des pluies. La courte saison pluvieuse s'étendant de début septembre à fin décembre parachève la boucle saisonnière du climat de cette relique forestière.

\section{Matériel}

Les Mones de Lowe sont des Primates diurnes. Leur régime alimentaire est composé de fruits, fleurs, feuilles et insectes. Ils récupèrent l'eau des feuilles, des branches et des trous dans les arbres (Bourlière et al., 1969). Deux groupes d'une population de Mones de lowe de 13 individus existent dans la relique forestière : un de cinq individus composés de deux mâles adultes et de trois femelles adultes, occupe préférentiellement la jachère, partie la plus grande de cette relique forestière. L'autre de huit individus est constitué d'un mâle adulte, quatre femelles adultes, deux subadultes et un juvénile, est inféodé dans la forêt secondaire. Ces deux groupes sont relativement habitués à la présence humaine pour permettre la collecte de données sur le suivi comportemental.

\section{Méthode}

\section{Suivi du groupe de singes}

Les méthodes d'observation comportementale ont concerné la population résiliente de Mone de Lowe Cercopithecus lowei Thomas, 1923, constitué par l'ensemble des 13 individus " habitués à la présence humaine ». Les méthodes utilisées sont celles du « scan sampling » et du focal animal sampling (Altmann, 1974). La collecte des données du suivi des groupes s'est faite pendant 24 mois soit 12 mois en 2018 et 12 mois en 2019. En général, le contact avec le groupe d'étude se faisait tôt le matin, vers 6 $\mathrm{h} 30$, heure à laquelle le groupe commençait à s'agiter. Il était alors possible de suivre ses déplacements à l'intérieur de la relique

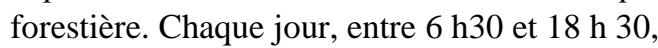
des observations périodiques par balayage du groupe suivi ont été faites (Altman, 1974) en utilisant des jumelles. Un balayage de $5 \mathrm{mn}$ était effectué toutes les 15 minutes, soit moyenne de 40 observations par jour et pour un total de 24417 «scans » pour toute la période d'étude. Au cours de ces 24417 balayages, des 
notes ont été prises sur chaque individu observé. Ces notes portent sur la classe d'âge de l'individu observé, l'activité en cours et éventuellement, l'item consommé (jeunes feuilles, feuilles matures, bourgeons, fleurs ouvertes, fruits non mûrs, fruits mûrs). Les notes collectées sur le positionnement dans la strate arbustive s'est référée sur la classification de la forêt de McGraw (1998a). Cet auteur a subdivisé les niveaux des arbres en strate 0 , strate 1 , strate 2 , strate 3 , strate 4 et strate 5. Quatre catégories d'activités ont été considérées : le fourragement, la locomotion, le repos et les interactions sociales (jeux, épouillage, accouplement). Les items alimentaires consommés ont concerné que le type d'organe végétal. Pour ces items, nous avons noté la nature (jeunes feuilles, feuilles adultes, bourgeons, fleurs, fruits non mûrs ou fruits mûrs), l'espèce de la plante dont il provenait, la saison, l'habitat et la strate arbustive.

Pour le focus, les deux groupes ont été suivis de façon alternée sur un intervalle d'une semaine. Seuls les individus adultes dont deux mâles et trois femelles d'un part et d'autre par un mâle et quatre femelles ont été observés pendant $5 \mathrm{~min}$ en fonction des groupes, trois fois par jour. Au total, nous avons réalisé 9015 focus. Chaque groupe a été suivi de façon continue et alternée au moins une fois par semaine suivant les différentes saisons de l'année. Lors du focus, ont été notés tous les comportements alimentaires individuels (choix de la nature de l'item végétal, conduite des congénères dans les habitats) et les évènements extérieurs « inhabituels » susceptibles d'entraîner un changement notable dans le comportement alimentaire de l'individu focal dont l'émission d'un cri.

Caractérisation floristique et phénologique des différents habitats

Lors du suivi comportemental des singes, des inventaires botaniques ont été effectués à partir de méthode des quadrats de sorte à identifier les espèces végétales et caractériser le type d'organes consommés dans la végétation dans les différents habitats de fourragement. Ainsi, sur un site d'arrêt des singes, nous délimitons un quadrat de $20 * 20 \mathrm{~m}$. L'arbre central dont le diamètre à hauteur de poitrine sera mesuré est marqué par du scotch (surtout la date), afin de collecter les données du quadrat. Cette démarche a été suscité dans le but d'éviter de déranger les singes et de biaiser les données à collecter. Dans ces quadrats de $20 * 20 \mathrm{~m}$, tous les arbres dont le diamètre à hauteur de poitrine $(d b h)>10 \mathrm{~cm}$, ont été mesurés et ceux dont la $d b h<10 \mathrm{~cm}$ dénombrés. Par ailleurs, des observations pilotes de 60 jours dans le site d'étude avaient permis relever et de positionner certains arbres fruitiers consommés par les Mones de Lowe étudiées. Ainsi, un total de 74 arbres fruitiers de toutes espèces confondues dont 16 dans la forêt secondaire, 57 dans la jachère et un arbre fruitier dans les espaces cultivés ont été géoréférencés. Un suivi d'observation mensuelle (début et mi- mois) par la méthode de la phénologie des arbres fruitiers a-t-elle permis de mettre en relief la disponibilité des items consommés dans les différents habitats (Goné Bi, 2007). Ce suivi a permis de caractériser la richesse des milieux le plus précisément possible sur les données phénologiques des arbres, arbustes et lianes. Nous nous sommes basés sur la proportion de la couronne occupée par les jeunes feuilles, les fleurs et les fruits des arbres, arbustes et lianes a été estimée en définissant cinq classes phénologiques : $0=0 \%, 1=] 0 ; 25 \%$ ], 2=] 25 ; 50\%], 3=] $50 ; 75 \%$ ] ,4=] $75 ; 100 \%$ ] (Koné et al.,2011).

\section{Analyse des données}

Nous avons d'abord déterminé la part des occurrences alimentaires des items d'espèces végétales qui ont été consommés. Puis, pour comparer la fréquence des items consommés, nous avons utilisé le test du Khideux $\left(\mathrm{x}^{2}\right)$ avec un risque d'erreur $\alpha=0,05$.

La richesse de la flore consommée a été déterminée par le dénombrement des espèces recensées sans tenir compte de leur abondance (Aké-Assi, 1984). Les différentes familles des espèces, ainsi que les types morphologiques ont été déterminés sur la base des listes d'espèces préétablies par Aké-Assi (2002). Ces 
paramètres ont permis d'apprécier la richesse et la composition floristique du répertoire alimentaire végétal des Mones de Lowe de la forêt de l'UNA. Le logiciel XLSTAT 2016 version 18.02.01.27444 a été utilisé pour la réalisation de tous les tests statistiques. La distribution des données ne suivant pas une Loi Normale, nous avons utilisé le test nonparamétrique de Kruskal-Wallis. Ce test a été utilisé pour comparer la quantité entre les types d'organes consommés dans les différents habitats, pendant les différentes saisons par ces animaux. Ensuite, le test de Dunn suivi de la correction de Bonferroni (avec 5000 permutations) (Mitchell et Takacs-Vesbach,
2008) a permis de regrouper ces items consommés. Pour déterminer la Préférence alimentaire d'origine végétale des Mones de l'UNA, nous avons utilisé l'analyse en composantes principales (ACP) pour vérifier la multicolinéarité entre les variables (Chatterjee et al., 2000).

Une Classification Ascendante Hiérarchique $(\mathrm{CAH})$ a été réalisée pour regrouper les 46 familles d'espèces végétales consommées par ces animaux. Les tests de Khi-deux $\left(\mathrm{x}^{2}\right)$ ont été réalisés pour comparer les différents effectifs entre les espèces recensées durant la période l'étude.

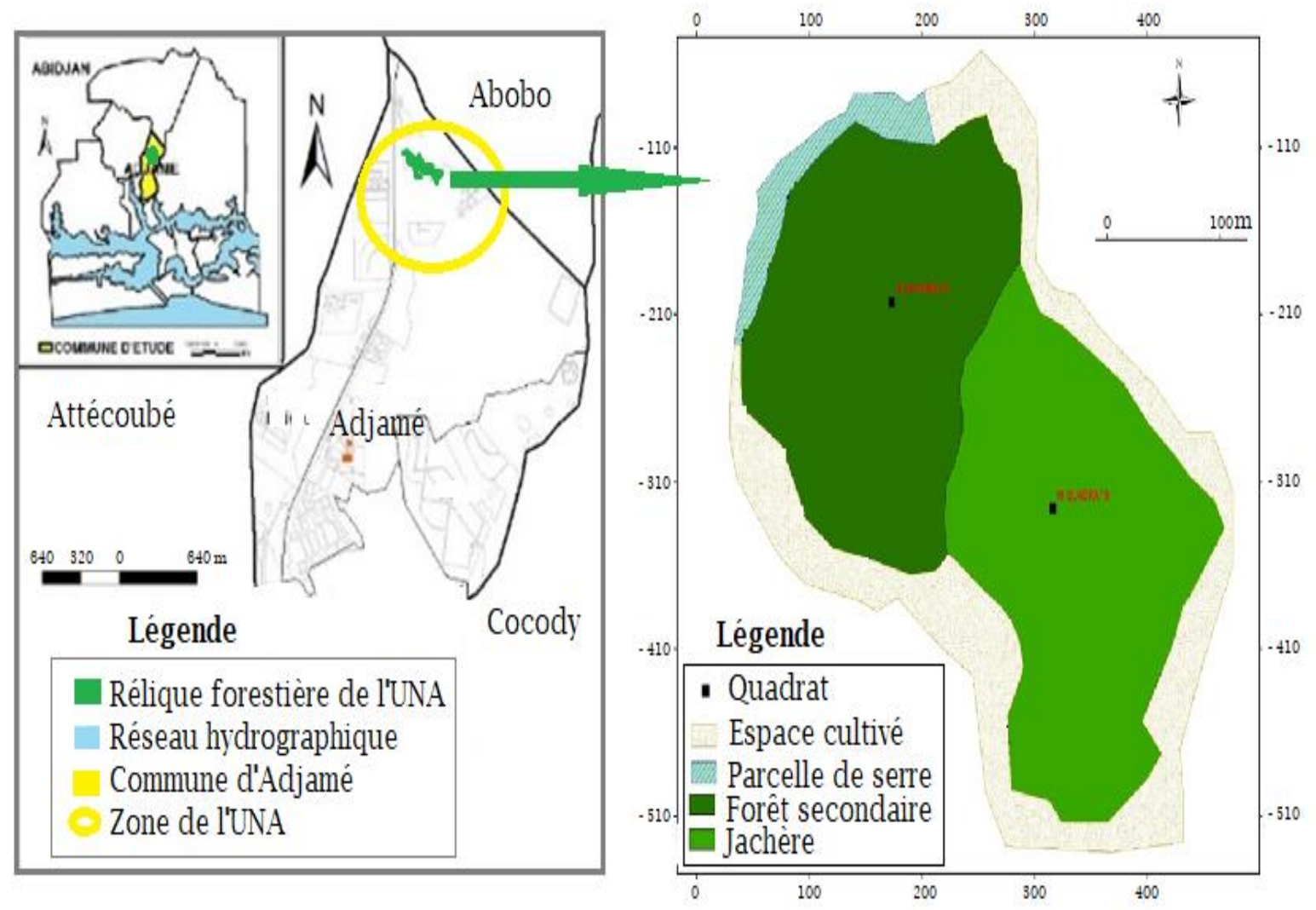

Figure 1 : Localisation de la forêt relique de l'Université Nangui Abrogoua. 


\section{RESULTATS \\ Diversité et Richesse de la flore consommée par les Mones de Lowe}

Les différentes observations ont permis de recenser 115 espèces végétales, exploitées comme sources de nourriture par les Mones dans l'UNA sur les deux années de l'étude. Elles se répartissent entre 79 genres et 46 familles. Le régime alimentaire des Mones de Lowe est dominé par 46 familles de plantes. La classification ascendante hiérarchique $(\mathrm{CAH})$ basée sur la distance eucludienne selon la méthode de Ward a permis de classer les 46 familles en trois groupes distincts (Figure 2). Le groupe 1 est celui des familles minoritaires mais ayant six à neuf espèces végétales consommées. Il est representé par les familles des Euphorbiaceae, des Annonaceae comptant huit espèces chacune et neuf espèces pour celle des Fabaceae. Le groupe 2 est celui des familles moyennes dont deux à cinq espèces végététales sont consommées. Il est representé par les familles des Apocynaceae, des Icacinaceae, etc.). Le groupe 3 est celui des familles majoritaires mais dont une espèce végétale est consommée. Il est representé par les familles des Aristolochiaceae, des Caricaceae, etc. (Figures 3). Cependant, le Test du $\mathrm{khi}^{2}$, p-value $=0,473>0,05$ n'a montré aucune différence significative entre le nombre de familles sur la durée de l'étude. Le nombre de familles était quantitativement identique sur les deux années (Tableau 1). Par ailleurs, ces espèces consommées sont réparties en deux grandes catégories à savoir les espèces indigènes (96 espèces soit $88,89 \%$ ) et les espèces introduites ou dites exotiques (12 espèces soit $11,11 \%)$. Les espèces indigènes les plus consommées sont Synsephalum afzelii, Chrysophyllum albinum, Psydrax subcordata, Parinari graba, Cleistophilis patens, etc. À ces espèces locales, s'ajoutent des espèces exotiques dont les plus fréquemment consommées sont Musa paradisiaca, Musa sapientum, Elaeis guineensis, Carica papaya, Manhiot escuelenta, etc.

\section{Type d'organes consommés par les Mones de Lowe}

Le répertoire alimentaire des Mones de Lowe de l'UNA est constitué de sept types d'organes de plantes (Figure 4) pour l'ensemble des 115 espèces végétales consommées. Le test de Kruskal-Wallis (pvalue $=0,001<0,05 ; \alpha=5 \%$ ) a montré une différence significative entre les types d'organes consommés. Les fruits sont consommés majoritairement à $79,4 \%$, les feuilles à $8,1 \%$, les fleurs à $6,3 \%$, à bourgeons à $3,6 \%$, les moelles à $2,7 \%$, les tiges, les racines ou tubercules et les champignons comptent chacun pour 0,9 .

Préférence alimentaire d'origine végétale des Mones de l'UNA

Les Mones ont consommé préférentiellement sur les deux années 30 espèces fruitières reparties sur l'ensemble des quatre saisons. Les deux premiers axes de la matrice de l'Analyse en Composantes Principales (ACP) (Figure 5) représentent $69,28 \%$ de la variation des données, avec des valeurs propres de $\lambda 1=1,67$ et $\lambda 2=1,11$, respectivement.

L'axe 1 de l'ACP, qui représente $41,56 \%$ de la variation est fortement corrélée aux régimes alinentaires des Mones durant les grandes saisons pluvieuses (GSP) et grandes saisons sèchesse (GSS).

Les choix des espèces végétales consommées par ces animaux pendant la GSP sont corrélés négativement durant GSS. Par contre, l'axe 2, qui exprime $27,72 \%$ de la variation est fortementlié aux types d'aliments consommés par les Mones durant les petites saisons sèches (PSS) et petite saison pluvieuse (PSP). Ainsi, les préférences alimentaires des Mones ont été réparties en trois groupes en prenant en compte les différentes saisons. Le groupe 1 représente l'ensemble des plantes consommé par les Mones pendant la GSS. Ce sont Chrysophyllum albinum, Parinari graba, Anthonotha macrophilla, Elaeis guineensis, Musa sp, Acacia mangium, etc. Quant au groupe 2, il est constitué des espèces de plantes telles que Maranga barteri, Manhiot esculenta, Salacia sp, Psydrax subcordata, etc., 
consommées par les singes durant les PSP et PSS. Le groupe 3 se caracterise par les végétaux comme Cleistophilis patens,Synsephaliun afzelli, Musa sp,polyalkia diveri, etc. que les Mones ont consommés pendant la GSP.

Distribution spatio- temporelle et disponibilité des items consommés par les Mones

La distribution spatiale des items tient compte de la position verticale (strate) et horizontale (habitat). Les données de cette distribution pour les deux groupes montrent qu'au niveau des habitats, les Mones de Lowe sont relativement inféodées à la forêt secondaire à 74,05\%, dans la jachère à 14,86\% et dans les espaces cultivés à 11,09\%. En outre, la recherche active de la nourriture par les Mones de Lowe montre que les individus sont présents sur toutes les strates arbustives. Les proportions dans l'occupation des strates sont fonction des saisons lors de la quête alimentaire. Le nombre des observations a montré que la distribution des items est inégalement répartie entre les différentes strates (Test du $\mathrm{khi}^{2}$, p-value $=0,0001<0,05$ ). Ainsi, durant la grande saison des pluies, le nombre total de «scans » était de 9996. Le Test de Procédure de Marascuilo ( $\mathrm{p}$-value $=0,0001<$ $0,05)$ a montré que les proportions d'occupation des strates ST3 $(34,47 \%)$, ST4 $(32,5 \%)$ sont majoritairement occupées au détriment des ST1 $(12,1 \%)$, ST2 $(17,67 \%)$ et ST0 $(3,33)$. Pendant, la grande saison sèche, le nombre total de «scan » était de 5703. Les strates ST4 $(39,53 \%)$, ST3 $(37,64 \%)$, ST1 $(13,40 \%)$ sont plus occupées que les strates ST2 (6,93\%) et ST0 (2,5\%). Cependant, durant la petite saison sèche où nous avions dénombré 4201 «scans ». Le Test de Procédure de
Marascuilo à p-value $=0,0001<0,05$ a montré que seule la strate ST2 $(46,58 \%)$ était plus exploitée par les singes que les trois autres ST1 $(15,63 \%)$, ST3 $(14,99 \%)$, ST0 $(14 \%)$ et la ST4 $(8,8 \%)$. Quant à la petite saison des pluies $(\mathrm{N}$ scan $=4015)$, les Mones, plus inféodées aux ST2 $(21,96 \%)$ et ST1 $(21,79 \%)$ et exploitaient moins les ST3 (15,39\%), ST0 (15\%) et faiblement la ST4 $(1,92 \%)$ (Tableau 2). Sur les 24417 scans valides dont 23815 observations d'occurrences alimentaires effectuées, l'analyse indique que la recherche des items d'origine végétale sont les ressources alimentaires les plus consommées $(68,02 \%)$ suivies de la consommation des invertébrés $(30,45 \%)$ et de la consommation d'eau $(1,52 \%)$ (Tableau 3). Suivant les saisons, durant la grande saison sèche, la comparaison par paire selon la Procédure de Marascuilo (pvalue $=0,0001<0,05$ ) montre que les Mones de Lowe consommaient plus les invertébrés $(63,2 \%)$. Pendant la grande saison des pluies, les végétaux étaient plus consommés $(47,4 \%)$ que l'eau $(25,5 \%)$ et les invertébrés $(8,6 \%)$. La petite saison sèche suit la même tendance que la grande saison des pluies. La petite saison des pluies est marquée une forte consommation d'eau $(35,2 \%)$ de végétaux $(11,1 \%)$ et des invertébrés $(10,1 \%)$. La quantité des items en terme richesse des sites en fonction des saisons a montré que ces données sont statistiquement différentes au seuil de significativité de $5 \%$ (Test de Kruskal-Wallis, p-value $=0,0001<$ $\alpha=5 \%$ ). La disponibilité des items est inégalement répartie entre les différentes saisons et dans les différents habitats selon le Test du khi ${ }^{2}, \quad$-value $=0,0001<\alpha=5 \%$. Il indique que la plus faible disponibilité était pendant la petite saison des pluies, suivie de la grande saison des pluies mai à août. 


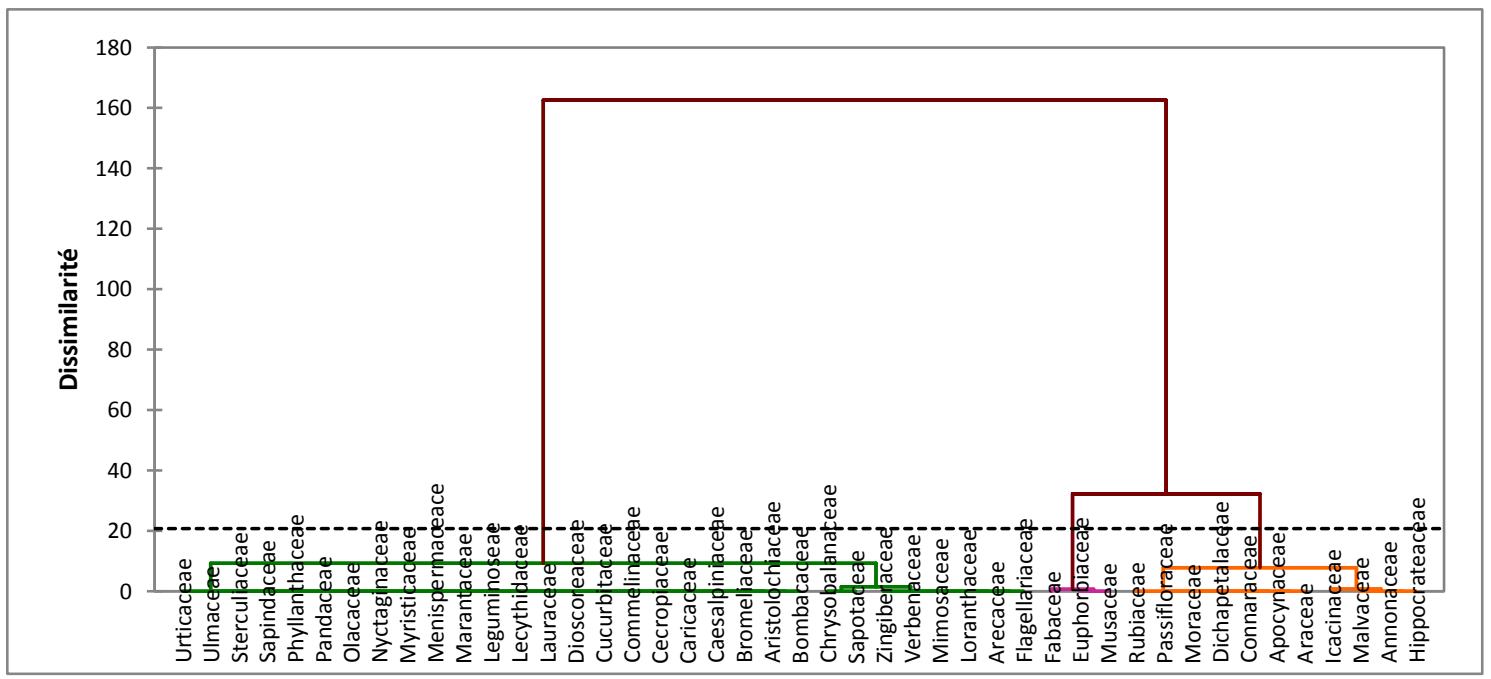

Figure 2 : Classification par famille des espèces de plantes en fonction de leur fréquence de consommation par le singe Cercopithecus lowei.

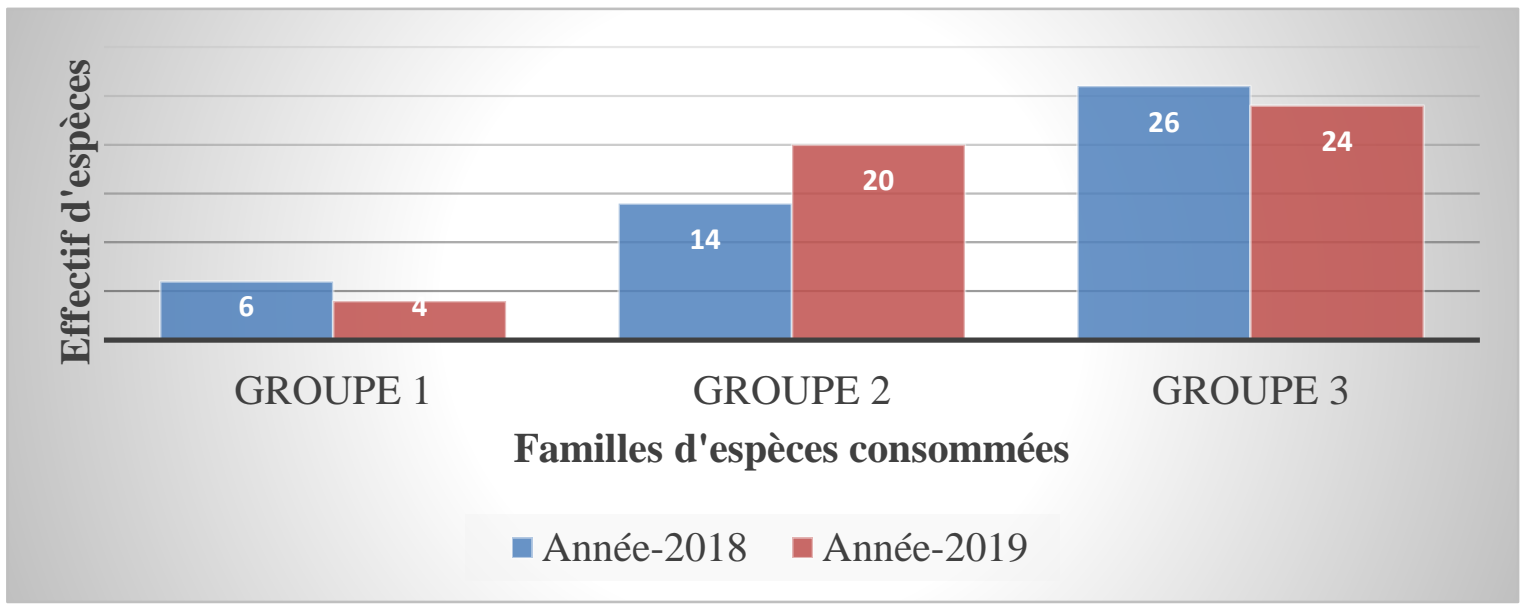

Figure 3 : Espèces végétales consommées par groupe de famille en 2018 et en 2019.

Tableau 1 : Comparaison des proportions d'espèces en fonction des saisons.

\begin{tabular}{lcc}
\hline Groupe de famille & Proportions-Année 2018 (\%) & Proportions-Année 2019 (\%) \\
\hline Groupe 1 & $60 \mathrm{a}$ & $40 \mathrm{a}$ \\
Groupe 2 & $41,2 \mathrm{a}$ & $48 \mathrm{a}$ \\
Groupe 3 & $52 \mathrm{a}$ & $58,8 \mathrm{a}$
\end{tabular}

Les proportions suivies d'une même lettre sont statistiquement identiques au seuil de 5\% (comparaison par paire de la Procédure de Marascuilo). 


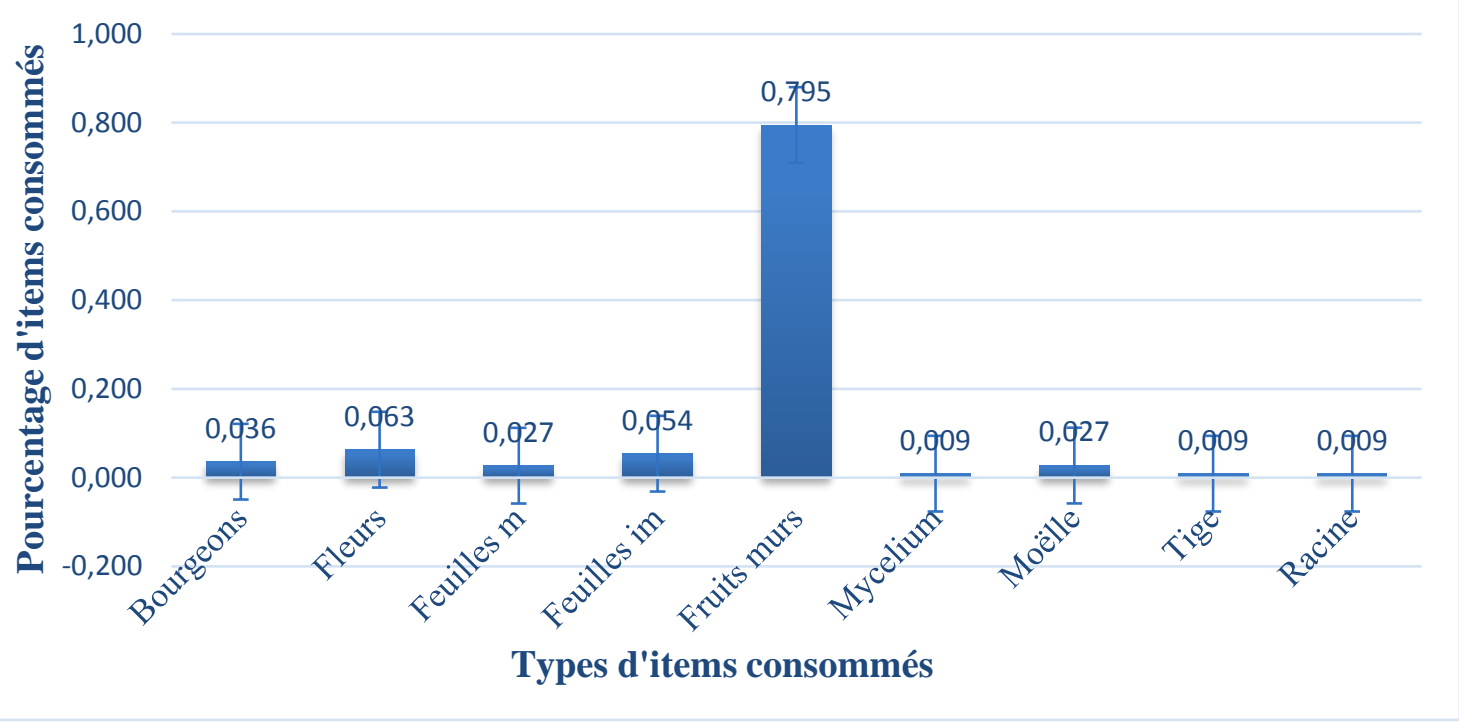

Figure 4 : Pourcentage des organes végétaux consommés par les Mones de l’UNA.

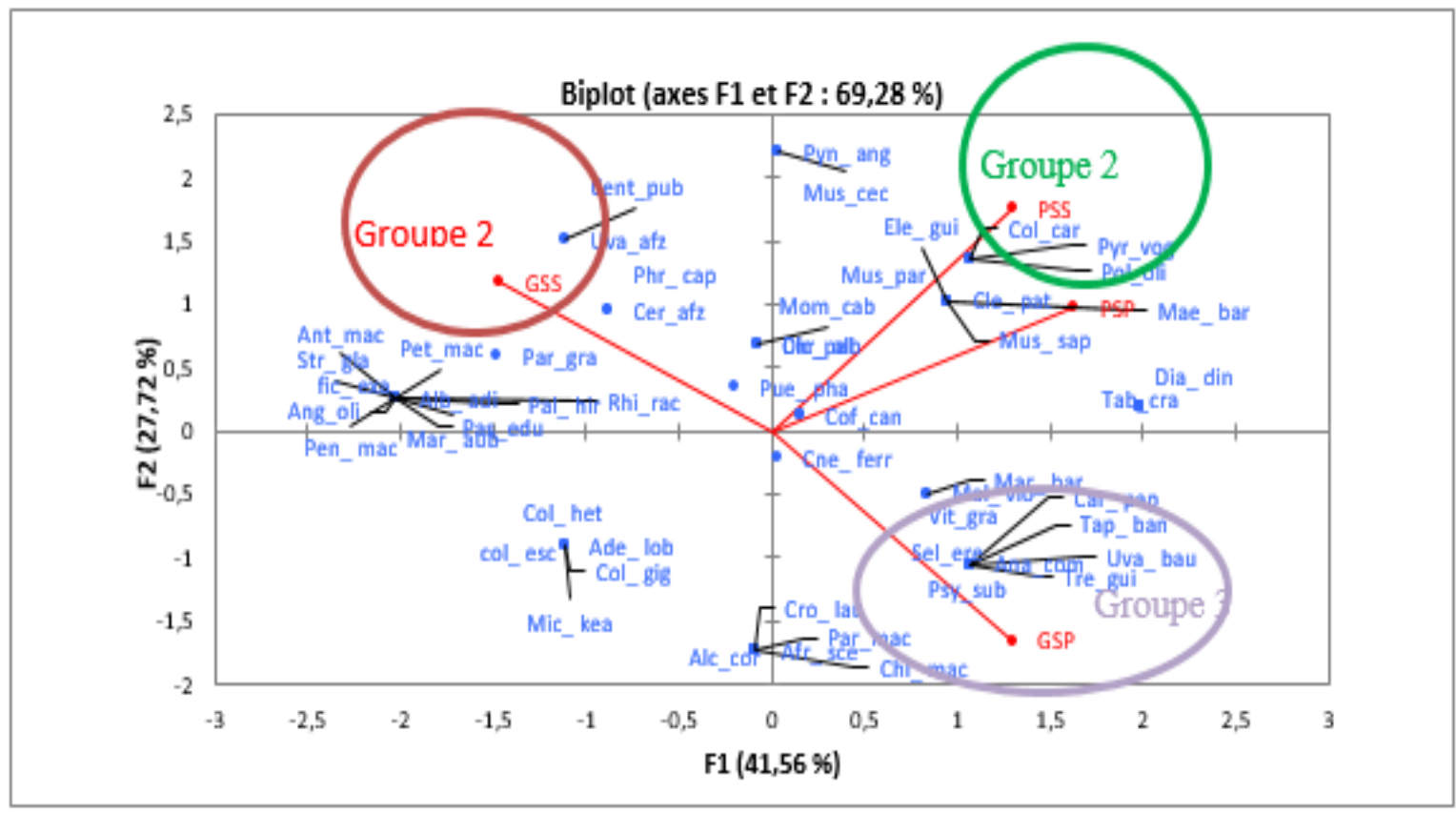

Figure 5 : Répartition des préférences alimentaires en fonction des 4 saisons (GSS, PSS, PSP et PSP) dans le plan cartésien F1 x F2 (69,28\% de la variabilité totale). 
Tableau 2 : Proportions des strates occupées par les Mones pendant les quatre saisons.

\begin{tabular}{lll}
\hline Saisons & Strates & Proportions (\%) \\
\hline Grande saison sèche & ST0 & $2,5 \mathrm{~b}$ \\
& ST1 & $13,4 \mathrm{c}$ \\
& ST2 & $6,93 \mathrm{a}$ \\
Grande saison des pluies & ST3 & $37,64 \mathrm{~d}$ \\
& ST4 & $39,53 \mathrm{~d}$ \\
& ST0 & $3,33 \mathrm{~d}$ \\
& ST1 & $12,1 \mathrm{a}$ \\
Petite saison sèche & ST2 & $17,67 \mathrm{~b}$ \\
& ST3 & $34,4 \mathrm{c}$ \\
ST4 & $32,5 \mathrm{c}$ \\
Petite saison des pluies & ST0 & $14, \mathrm{~b}$ \\
& ST1 & $15,5 \mathrm{~b}$ \\
& ST2 & $46,5 \mathrm{c}$ \\
& ST3 & $15 \mathrm{~b}$ \\
& ST4 & $8,8 \mathrm{a}$ \\
ST0 & $19 \mathrm{~b}$ \\
Les proportions suivies d'une même lettre sont statistiquement identiques au seuil de & $5 \%$ (Test de Procédure de Marascuilo) \\
pendant les différentes saisons. & & $28,8 \mathrm{c}$ \\
\end{tabular}

Tableau 3 : Comparaison des proportions des ressources alimentaires en fonction des saisons

\begin{tabular}{lcccc}
\hline Items & $\begin{array}{c}\text { Proportions } \\
\text { GSS }(\boldsymbol{\%})\end{array}$ & $\begin{array}{c}\text { Proportions GSP } \\
(\boldsymbol{\%})\end{array}$ & $\begin{array}{c}\text { Proportions } \\
\text { PSS }(\boldsymbol{\%})\end{array}$ & Proportions PSP (\%) \\
\hline Item végétal & $24,3 \mathrm{a}$ & $47,4 \mathrm{c}$ & $17,2 \mathrm{~b}$ & $11,1 \mathrm{a}$ \\
Item animal & $70,9 \mathrm{~b}$ & $8,6 \mathrm{a}$ & $10,4 \mathrm{a}$ & $10,1 \mathrm{a}$ \\
Autres & $28,3 \mathrm{a}$ & $25,5 \mathrm{~b}$ & $11 \mathrm{a}-\mathrm{b}$ & $35,2 \mathrm{~b}$ \\
\hline
\end{tabular}

Les proportions suivies d'une même lettre sont statistiquement identiques au seuil de 5\% (comparaison par paire de la Procédure de Marascuilo).

\section{DISCUSSION}

Le répertoire alimentaire des Mones de Lowe de la forêt de l'UNA compte 115 espèces végétales. Ce répertoire est relativement diversifié du fait de la réduction de la superficie de la relique forestière. Cette réduction a eu pour conséquence la disparition d'une bonne partie des essences végétales de cette relique, d'où la réduction les ressources alimentaires de ces singes. Cependant, il est plus élargi que celui de trois espèces sympatriques de cercopithèques à savoir la Mone de campbell, le cercopithèque nez- blanc et le cercopithèque diane du Parc National de Taï en Côte d'Ivoire (Buzzard, 2006, 2010). En effet, ses trois espèces avaient consommé entre 95 et 105 espèces de plantes. Au Parc National de Kankum au Ghana, le répertoire alimentaire de 
la Mone de lowe était estimé à 36 espèces de plantes fruitières (Edward, 2015). Dans la réserve nationale de Kibale, en Ouganda, 325 espèces consommées par Cercopithecus mitis, ont été recensées par (Worman et Chapman, 2006).

La grande diversité des espèces et des items consommés par les Mones dans un tel fragment de forêt montre leur plasticité alimentaire pour pallier la disponibilité des ressources fluctuantes. En effet, selon les études de Milich et al. (2014) et Dunham (2017), les PNH des fragments de forêt compensent la disponibilité de la ressource alimentaire en augmentant le temps de fourragement et la diversité des items consommés. Cette grande diversité alimentaire pourrait s'expliquer par la diversité de la flore des habitats qui composent la relique (Missa et al., 2019). En effet, la relique est très perturbée par les activités anthropiques. Ainsi, l'on rencontre la forêt secondaire et des champs de cultures de subsistance entretenus. Outre ces deux habitats, il y a la jachère, dominée par Acacia mangium et des espèces lianescentes. Dans un tel petit habitat dégradé et pauvre en qualité, la flexibilité alimentaire consiste à avoir une large gamme des items (Donati et al., 2011). Tous ces biotopes offrent une plus grande variété d'espèces dont la concentration est la plus élevée en forêt secondaire et dans la jachère, deux habitats relativement identiques en diversité spécifique. Le régime alimentaire des Mones de Lowe est polymorphe et est constitué de plusieurs types de plantes et d'organes végétaux rencontrés dans les différents habitats de la relique de l'UNA. Dans la forêt secondaire (FS), les organes d'espèces arborescentes indigènes, exotiques (Musa sapientium,Carica papaya à la limite de la FS) et lianescentes sont les plus consommés par ces singes. Dans la jachère, les espèces lianescentes de Salacia $s p$ pendant la petite saison des pluies, les espèces arborescentes exotiques (Acacia mangium) pendant la grande et la petite saison sèche, sont plus consommées. Quant à Elaeis guineensis, elle est majoritairement consommée pendant la grande saison sèche. Des observations similaires ont été faites par Kouakou (2019) et Koné et al. (2011). Les résultats de cette étude relèvent qu'en plus des espèces arborescentes, les espèces lianescentes constituent le groupe d'espèces les plus consommées par les Mones de l'UNA. Les travaux d'Edward (2015) au Ghana et Kouakou (2019) en Côte d'Ivoire ont montré que les Mones ont une appétence pour les cultures de subsistance. Ce qui confirme notre étude, lorsque les Mones de lowe font des incursions dans les champs de cultures vivrières à la recherche de Musa sp et de Manihot esculenta. Les incursions de ces Mones à la recherche de ces items particuliers causent de graves dégâts pour les planteurs et entrainent des conflits homme-faune (Kouao et al., 2017). Pour certains auteurs, le régime alimentaire des Primates non- humains en général et en particulier, entre espèces très voisines des cercopithèques (Mone de Lowe et Mone de campbell) varie en fonction des saisons (Buzzard, 2010).

Zausa et al. (2018) a montré que l'essentiel de la nourriture de la Mone de Campbell, espèce très voisine de la Mone de Lowe était constitué en grande partie d'invertébrés pendant la grande saison sèche dans le Parc National de Taï, (Côte d'Ivoire). Buzzard (2010), dans son étude a montré dans ce même Parc et sur la même espèce voisine de la Mone de Lowe que l'essentiel de la nourriture était constitué en grande partie de fruits pendant les saisons de pluie.

Les Mones de Lowe sembleraient recourir à un large éventail d'items pour avoir des nutriments nécessaires indispensables à leur survie. Selon Donati et al. (2015) les primates des milieux dégradés modifient leur comportement alimentaire pour recueillir le maximum d'énergie contenue dans chaque unité d'item.

De plus, les primates de ces milieux compensent leur anatomie physiologique nonspecialisé -avec une diversité des items pour accroître les ressources alimentaires durant les périodes de moindre disponibilité (Campera et al., 2014 ; Donati et al., 2015).

Durant les saisons qui coïncident avec la rareté des fruits des espèces indigènes, les 
primates peuvent consommer en grande quantité les fruits exotiques (Colquhoun, 1998). Cette étude a permis de montrer que les espèces Musa sp, Elaeis guineensis, Acacia mangium, etc. étaient préférentiellement consommés par les Mones de lowe pendant les saisons sèches.

La présente étude sur les Mones de l'UNA relève l'existence de Musa sp dans l'ensemble de la flore de régime alimentaire de ces singes. Cette espèce végétale, très abondante dans les espaces cultivés, était parmi les 30 espèces les plus consommée dans la zone d'étude. Koné et al. (2011) avaient conclu ces singes parcouraient de grande distance souvent en dehors de leur territoire pour les ressources privilégiées. Kouao et al. (2017) et Kouakou (2019) ont montré que les dégâts causés par les Cercopithèques dans les zones périphériques des Parc Nationaux et les Mones dans les forêts villageoises deviennent très importants dès que les cultures arrivent à maturité. Ces spéculations sont généralement représentées par des cultures vivrières (bananiers, manioc) et des cultures pérennes (cacaoyers). Les résultats obtenus dans cette étude montrent que les Mones ont des préférences alimentaires qui ne tiennent pas forcément compte des habitats. En effet, l'on a observé que le type d'organe prélevé variait d'une espèce à l'autre et d'un habitat à un autre. Cela suggère qu'ils ont une bonne connaissance de la phénologie des espèces végétales qui composent ce régime alimentaire (Koné et al., 2011).

Parmi les différents organes recensés dans cette étude, les fruits sont les plus abondants. L'importance des fruits dans le régime alimentaire des Mones pourrait se justifier par sa disponibilité quasi permanente sur toute l'année. Des travaux réalisés sur la nature du régime alimentaire des Cercopithèques forestiers ont abouti à des résultats similaires (Buzzard, 2006). Selon les études effectuées par Buzzard (2010) dans le Parc national de Taï, 75 à 105 espèces de plantes sont consommées pour leurs fruits. Parmi les espèces fruitières de la forêt de l'UNA, Synsephalium afzelli (Apocynaceae), Psdrax subcorda (Rubiaceae) et Acacia mangium (Fabaceae) étaient parmi les cinq premières espèces de plantes les plus consommées par ces singes à queue. Les fruits représentent $79,6 \%$ des items d'origine végétale consommée par les Mones de Lowe dans la relique de l'UNA.

L'importance des fruits dans le régime alimentaire des singes à queue avait déjà été signalée par de nombreux auteurs en Afrique de l'Ouest (Koné et al., 2008). Koné et al. (2011) avaient recensé 34 espèces végétales dans la même relique forestière de l'UNA dont les fruits sont préférentiellement consommés. Les 30 espèces végétales préférentiellement consommées et mises en évidence dans cette étude sont relativement en deçà des résultats de Koné et al. (2011). Cela s'explique par le fait que la relique forestière a subi la destruction d'une grande partie de sa surface qui comptait les ressources végétales alimentaires des mones. Cette surface ayant servi à la construction des infrastructures universitaires, a eu pour corollaire la réduction d'une bonne partie des ressources végétales des Mones de lowe. Ce nombre est aussi inférieur à une liste de 36 espèces de fruits qui fut dressée au Ghana (Edward, 2015).

Les fruits consommés par les Mones de l'UNA sont dans leur grande majorité de petite taille, capable d'être stockés dans leurs bajoues puis être consommés dans les conditions idéales. Ces fruits appartiennent à des espèces de toutes les strates des habitats et sur les branches pouvant supporter leur poids relativement léger. En tenant compte de la flexibilité alimentaire de ces Mones de Lowe et de l'armature buccale dentaire, Wiafe (2016) et Bourlière et al. (1976) affirment que les Mones de Lowe mangent tous les fruits charnus qu'ils trouvent à condition que le fruit contienne la valeur nutritive recherchée. Comme exemples, nous pouvons citer Synsephalium afzelii, Cleistophilis patens, Acacia mangium, Tabernaemontana crassa, etc. Selon Koné et al. (2011), ces Mones de l'UNA peuvent aller au-delà de leur territoire pour rechercher les items les plus appétés. Comme exemples, nous pouvons citer Musa sapientium, Carica papaya, Manhiot escuelent,qui se trouvaient 
dans les parties cultivées. Elaeis guineensis, Strombosia glaucoscens et Dichapetalium filicaule rencontrés dans la partie jachère et dans la partie forêt secondaire, étaient convoités par chaque groupe. Dans cette relique forestière de l'UNA, les Mones de lowe consomment dans la forêt secondaire, habitat sûr pour fuir les prédateurs, les fruits des grands arbres. Ces espèces végétales de cette partie de la relique, telles que Parina graba, Synsephalium afzelii, Chrysophyllium albinum, Cleistophilis patens disposant donc des meilleures structures et produisant beaucoup de fruits, assurent la survie de cette population résiliente des Mones.

\section{Conclusion}

Cette étude a montré que le répertoire alimentaire des Mones de Lowe de l'UNA est très diversifié. Il comprend 115 espèces végétales appartenant principalement à la famille des Apocynaceae Euphorbiaceae, Ceasalpinaceae, Annonaceae, Musaceae, Arecaceae et Rubiaceae. Au sein de ces familles, certaines espèces comme Cleistophilis patens, Elaeis guineensis, Musa sp, Psydrax subcordata, Synsephalium afzelli, Acacia mangium, Parina graba, Chrysophyllum albinum, Dichapetalum Phragmenthera capitata, Tapinanthus bangwensis apparaissent plus consommées. Sur ces différentes espèces recensées, les Mones de lowe prélèvent sept types d'organes principalement les fruits, les tubercules et les fleurs. Dans cette flore, l'on note une relative dominance des items dans la jachère que la forêt secondaire et les champs. Pendant les petites saisons, les basses strates de la jachère offrent une diversité d'items au Mones. Quant à la forêt secondaire, occupée par des grands arbres, leurs hautes strates 3 et 4 hébergent, au cours dans les grandes saisons, des fruits et des invertébrés, beaucoup consommés par ces Mones. Des études supplémentaires exigées permettront d'évaluer la valeur nutritive des items consommés afin de connaître leur potentiel énergétique ce qui permettra d'assurer la survie des Mones de lowe de l'UNA.

\section{REMERCIEMENTS}

Nous remercions les autorités de l'UNA et en particulier les Doyens et vice -doyen de l'UFR des Sciences de la Nature de ladite université pour avoir autorisé cette étude. Nous voulons dire un grand merci au Directeur Centre Suisse de Recherches Scientifiques qui a mis à notre disposition le matériel. Nous remercions également tous les chercheurs qui ont contribué à la relecture de versions antérieures de cet article. Nous oublierons de dire grand merci à M. TERE Henri, a qui contribué à la récolte des données et à l'identification des espèces de plantes.

\section{REFERENCES}

Aké ASSI L. 2002. Flore de la Côte d'Ivoire : catalogue systématique, biogéographie et écologie. Mémoire de Botanique systématique. Boissiera 58 Tome II, 401p.

Aké ASSI L. 1984. Flore de la Côte d'Ivoire : étude descriptive et biogéographique, avec quelques notes ethnobotaniques Tome I et II. Thèse Doctorat. Ès Sciences de la Nature. FAST, Université d'Abidjan-Cocody. 1205 p.

Altmann J. 1974. Observational study of behavior: Sampling methods. Behaviour, 49: 227-266.

Asare R, Afari-Sefa V, Osei-Owusu, Y, Pabi O. 2014. Cocoa agroforestry for increasing forest connectivity in a fragmented landscape in Ghana. Agroforest Syst. DOI: 10.1007/s10457014-9688-3. Published online 29 March, 2014

Avenard JM, Eldin M, Girad G, Sircoulon J, Touchebeuf P, Guillaumet J, Adjanohoum E, Perraud A. 1971. Le milieu naturel de Côte d'Ivoire. Mémoire ORSTOM n ${ }^{\circ} 50$, Paris Franc, 1-392.

Bamba K, Béné JCK, Célestin YK, Kouamé AN, Victorien K.C. 2017. Diversité, distribution et statut de conservation des primates dans les reliques de forêts dans la région du Tonkpi, à l'ouest de la Côte d'Ivoire. European Scientific Journal, 13(26) : 20-41. 
Bitty EA, Gonedele B, Koffi BJC, Kouassi KP, McGraw WS. 2015. Cocoa farming and primate extirpation inside Cote d'Ivoire's protected areas. Tropical Conservation Science, 8(1) : 95-113, 2015.

Bourlière F, Bertrand M, Hunkeler C. 1969. L'Ecologie de la Mone de Lowe en Côte d'Ivoire. Terre Vie, 23: 135-163.

Buzzard PJ. 2010. Polyspecific associations of Cercopithecus campbelli and C. petaurista with C. diana: what are the costs and benefits? Primates, 51: $307-$ 314.

Buzzard PJ. 2006. Ranging patterns in relation to seasonality and frugivory among Cercopithecus Campbelli,C. petaurista and $C$. diana in the Taï forest. International Journal of Primatology, 27: 559-573.

Campera M, Serra V, Balestri M, Barresi, M, Ravaolahy M, Randriatafika F, Donati G. 2014. Effects of habitat quality and seasonality on ranging patterns of collared brown lemur (Eulemur collaris) in littoral forest fragments. International Journal of Primatology, 35: 957-975.

Colquhoun IC. 1998. Cathemeral behavior of Eulemur macaco macaco at Ambato, Massif, Madagascar. Folia Primatologica, 69 (1): 22-34.

Chatterjee, Samprit A S, Hadi B, Price. 2000. Regression analysis by example. John Wiley \& Sons Inc.: New York.

Donati G, Campera M, Balestri M, Serra V, Barresi M, Schwitzer C, Curtis DJ, Santini L. 2015. Ecological and anthropogenic correlates of activity patterns in Eulemur. International Journal of Primatology. DOI: 10.1007/s10764-015-9876-7

Donati G, Kesch K, Ndremifidy K, Schmidt SL, Ramanamanjato JB, BorgogniniTarli SM, Ganzhorn JU. 2011. Better few than hungry: flexible feeding ecology of collared lemurs Eulemur collaris in littoral forest fragments. PLOS ONE, 6: e19807.

Dunham NT. 2017. Feeding Ecology and Dietary Flexibility of Colobus angolensis palliatus in Relation to Habitat
Disturbance. International Journal of Primatology, 38(3). DOI: 10.1007/s10764-017-9965-x

Edward DW. 2015. Nutrient contents of three commonly consumed fruits of lowe's monkey (Cercopithecus campbelii lowei). Springerplus, 4: 44.

Estrada A, Garber P A, Rylands AB, Roos C, Fernandez-Duque E, Di Fiore A, Nekaris KAI, Nijman V, Heymann EW, Lambert JE, Rovero F, Barelli C, Setchell JM, Gillespie TR, Mittermeier RA, Arregoitia LV, Miguel de Guinea, Gouveia S, Dobrovolski R, Shanee S, Shanee N, Boyle SA, Fuentes A, MacKinnon K C, Amato K. R, Meyer ALS, Wich S, Sussman RW, Pan R, Kone I, Baoguo. 2017. Impending extinction crisis of the world's primates: Why primates matter, American Association for the Advancement of Science.

Goné Bi ZB. 2007. Régime alimentaire des chimpanzés, distribution spatiale et phénologie des plantes dont les fruits sont consommés par les chimpanzés du Parc National de Taï, en Côte d'Ivoire, Thèse de l'Université de Cocody-Abidjan Spécialité : Ecologie Végétale $174 \mathrm{p}$

Koné I. 2015. Community empowerment for the conservation of critically endangered primates and their habitat in SouthEastern Côte d'Ivoire. In Twenty-two Stories of Conservation in Africa: key Elements for Effective and Well-governed Protected Areas in Subsaharan Africa. IUCN PAPACO; 36-39.

Koné I, Bahans LI, Ouattara K. 2011. Les mones de Lowe (Cercopithecus campbelli lowei) utilisent une stratégie de fourragement optimale dans la forêt Réserve de l'Université d'AboboAdjamé, Côte d'Ivoire. International Journal of Biological and Chemical Sciences, 5(3): 1265-1277.

Koné I, Lambert JE, Refisch J, Bakayoko A. 2008. Primate seed dispersal and its potential role in maintaining useful tree species in the Taï region, Côte-d'Ivoire: implications for the conservation of forest 
fragments. Tropical Conservation Science, 1(3): 293-306.

Kouadio KP, Yaokokoré, BKH, Odoukpé, KSG, Konan EM, Kouassi K P. 2014. Diversité Avifaunique du Parc National du Banco, Sud-Est Côte d'Ivoire. European Journal of Scientific Research, 125(2) : 384-398.

Kouakou CV. 2019. Importance des fragments de forêt dans la conservation des primates non-humains en Côte d'Ivoire : cas de la forêt sacrée et des forêts villageoises à Gbétitapéa dans la région du HautSassandra, Thèse de Doctorat 155p, soutenue publiquement à l'Université Jean LOROUGNON GUEDE-DaloaCôte d'Ivoire

Kouao M L, Bené JCK, Koffi AD, Kouamé B A, Koné I. 2018. Caractérisation des dégâts provoqués par la faune sauvage à la périphérie de la Forêt des Marais Tanoe-Ehy au sud-est de la Côte d'Ivoire, International Journal of Biological and Chemical Sciences, 12 (4) : 1717-1730.

McGraw WS.1998a. Comparative Locomotion and Habitat Use of Six Monkeys in the Tai Forest, Ivory Coast. American Journal of Physical Anthropology, 105: 493-510.

Lauginie F. 2007. Conservation de la nature et des aires protégées en Côte d'Ivoire. NEI/ Hachette et Afrique Nature, Abidjan, 1668.

Milich KM, Stumpf RM, Chambers JM, Chapman CA. 2014. Female red colobus monkeys maintain their densities through flexible feeding strategies in logged forests in Kibale National Park, Uganda. American Journal of Physical Anthropology, 154: 52-60.

Mitchell, Kendra R, Cristina DTV. 2008. A comparison of methods for total community DNA preservation and extraction from various thermal environments. Journal of Industrial Microbiology \& Biotechnology, 35(10): 1139-1147.
Missa K, Soro D, Seguena F, Bakayoko A. 2019. Floristic and structural studies of the forest blocks of Nangui Abrogoua University, Cote d'Ivoire. Journal of Biodiversity and Environmental Sciences, 14(1): 61-71.

Olupot W. 1994: Ranging Patterns of the GreyCheeked Mangabey Cercocebus albigena with Special Reference to Food Finding and Food Availability in Kibale National Park, M.Sc. Thesis, Makerere University, Kampala.

Wiafe ED, Oates, JF, Gonedelé Bi S, Koné I, Matsuda Goodwin, R, Osei D. 2019. Cercopithecus lowei. The IUCN Red List of Threatened Species on www.iucnredlist.org. http://dx.doi.org/10.2305/IUCN.UK.201 9

Wiafe ED. 2016. Population studies of Lowe's monkey (Mammalia: Primates: Cercopitecidae: Cercopithecus lowei Thomas, 1923) in Kakum Conservation Area, Ghana. Journal of Threatened Taxa, 8(2): 8434-8442.

Worman CO, Chapman CA. 2006. Densities of Two Frugivorous Primates with Respect to Forest and Fragment Tree Species Composition and Fruit Availability. International Journal of Primatology, 27(1).

Yeo K, Tiho S, Ouattara K, Konate S, Kouakou LMM, Fofana M. 2013. Impact de la fragmentation et de la pression humaine sur la relique forestière de l'Université d'Abobo-Adjamé (Côte d'Ivoire). Journal of Applied Biosciences, 61: 4551-4565.

Zausa D, Koné I, Ouattara K. 2018: The optimal foraging strategy used by Campbell's Monkeys, Cercopithecus campbelli, in the dry season in the Taï National Park (Côte d'Ivoire). International Journal of Research in BioSciences, 7(4): 8-20. 